\title{
Eric B. Winsberg (2018) Philosophy and Climate Science. New York: Cambridge University Press. 270 pages. ISBN 978-1-316-64692-2
}

\author{
José Luis Granados Mateo \\ jlgranados.ing@gmail.com
}

During the last two decades, numerous books have been written from philosophy in relation to climate change. Most of them concern ethical, social, and/or political issues. However, this book by Eric Winsberg provides a general approach to Climate Science from an eminently epistemological perspective. Following the publication of his earlier work, Science in the Age of Computer Simulation (2010), Winsberg offers an introduction to the Philosophy of Climate Science that aims to contribute-as the author himself states - to a deeper understanding of the general public to the scientific practices that currently influence environmental policy. Although the book is aimed at philosophers of science in other areas, I believe that this book could be especially valuable for scientists and technologists researching in fields where philosophy is apparently far away. For climatologists, oceanographers, atmospheric physicists, or any scientist directly or indirectly involved in the study of climate change, reading this book can provide a deeper insight into the kind of knowledge applied in everyday scientific research. And, conversely, the philosophers of science who deal with classical questions will see how old problems are revived in these sciences whose practices are remarkably new.

The philosophy of climate science is interested in understanding the logic, methodology, and conceptual underpinning of these sciences within a broad and consistent epistemological framework. Some of the philosophical questions that Winsberg addresses throughout the fourteen chapters that structure the book are: the nature of scientific data and its relation to theory, the role of computational models and simulations, the character of probabilities in science and decision-making, statistical inferences, the influence of ethical and social values in scientific practice, the social processes of knowledge construction, etc. Most of these contain a compendium of the research that Winsberg has published throughout the last decade, so it can also function as an introductory manual to one of the most fruitful researchers in the philosophy of climate science. In what follows, I will explore the contents of the book which are, arguably, the most illuminating for the scientific community and philosophers of science in general.

Winsberg begins the second chapter by addressing the observational data-collected through weather stations and satellites-that we already know about the climate. It also addresses the hypotheses that have been generated based on this information, stating that, indeed, the climate has already changed significantly over the last century. He first warns that these hypotheses are inferred from what Patrick Suppes (1969) called Models of Data; a reconstruction of the raw data collected by thousands of thermometers, sensors, weather balloons, and a series of instruments distributed around the planet, which reexamines the Dunhem/Quine problem and the need to use 'auxiliary hypotheses' when making scientific predictions. The central question defended by Winsberg is the epistemic validity of these models 
against critics who try to invalidate techniques that actually require great scientific sophistication and the cooperation of a plurality of experts. A case of what, in philosophical literature, is called 'confirmational holism' which is not exclusive to the sciences of climate change, but present in well-established physical theories (Dunhem, 1991), and even in logic and mathematics (Quine, 1953).

Chapters three and four deal with climate modeling, with an emphasis on Energy Balance Models (EBMs), but also on Models of Mediation. Likewise, he reviews the epistemology of scientific simulations, which is the main theme of his previous book (Winsberg, 2010). Chapter five is dedicated to the chaotic nature of atmospheric dynamics and its scientific implications. In particular, he explains the difference between making predictions in meteorology and making projections in climatology, providing a rigorous conceptual clarity to what is an otherwise chaotic system, and by demarcating the role of the Butterfly Effect phenomenon in each case. Through the example of two kinds of simple chaotic models-Lorentz's model and Robert May's logistic application-he illustrates several features which are relevant when philosophically studying climate systems. Winsberg emphasizes that, even in a chaotic system, the degree of predictability depends largely on the initial conditions introduced and, once again, on the type of prediction scientists want to make. Through examining the strategies with which they try to mitigate the butterfly effect-such as the PICEF, a type of forecast in which a point prediction for the state of the system in the future is replaced by a distribution over possible future states-and by drawing a comparison with the Hawkmoth Effect, Winsberg concludes that there is no analogous model close to the structure of the butterfly effect model, and even if there were, it would not affect climate projections. Precisely because they are not predictions, but 'forced experiments', in which we change the initial conditions to study one or another aspect of the system.

The second part of the book deals with the role of probability and Uncertainty Models. Chapter six focuses on the role of Uncertainty Models in the communication of (dis)knowledge from experts to politicians, being the method with the least subjective bias available. Unlike other sciences, climate sciences have a significant social and political influence, and although scientists are well-suited to talk about how the climate will behave, they do not represent the values and interests of society. Thus causing Winsberg, in the next chapter, to question the confidence placed in these models. He assembles in seven categories the sources of uncertainty that influence the allocation of probabilities in each of the possible scenarios proposed by the IPCC. Winsberg explains that these probabilities are a representation of beliefs, not an exact mechanistic quantification. They are estimates that include unquantifiable factors, such as the experience of scientists, and represent the consensus of a group of experts, which is one more objective that scientists must satisfy.

Chapter eight deals with statistical inferencefocusing on the Bayesian paradigm and the frequentist-and decision-making under uncertainty. It also includes a discussion on Integrated Assessment Models (IAMs), in which Winsberg warns that they should not be presented as a 'scientific alternative' to political decision-making based on ideology. To use them responsibly, they need to take into account basic elements such as Equilibrium Climate Sensitivity (ECS) and Damage Function, which could significantly influence their results. Following this thread, chapter nine begins by asking to what extent scientific research can-and should-reflect the social and ethical values that surround it. For its part, it seeks to clarify what role these play in the case of climate science, distinguishing between the traditional context of discovery and the context of justification. Winsberg evokes the famous debate between Richard Rudner and Richard Jeffrey on the argument of inductive risk, in order to demonstrate how climate modeling advocates ought to accept the inevitable role of values in science.

In chapter ten, Winsberg focuses on the skill of models; a quality that identifies it with the capacity of models to obtain adequate results with respect to the purposes for which they have been built. Discussions are included about the verification and validation of these models, and it is concluded that these processes are 
rarely useful in the case of climate modeling. Rather than following a set of normative rules, the skill of these models concerns small ad-hoc modeling, so Winsberg advocates leaving aside such rules. However, when dealing with the reliability of inferences derived from a tuned simulation, a general pragmatic criterion should still be observed: if a simulation is capable of successfully predicting new data, then it is reliable. In this way, it assigns the value of consequences before the way in which the causative modeling of results is configured, which may depend on the epistemic positioning of the evaluator. This leads him to consider, in chapters eleven and twelve, the role of robustness analysis; that is, on the epistemological value of hypotheses arising from the integration of a set of models, which can be supported by a greater or lesser amount of evidence. For example, if several different models demonstrate that we should expect the ice caps to melt at this or that speed, is there more evidence if additional models accumulate, indicating the same? To this end, Winsberg reviews the properties that models should have so that there is sufficient diversity to obtain epistemologically robust hypotheses.

Chapter thirteen introduces a theme that, in my opinion, is one of the most revealing of the whole book: the social epistemology in climate science. Specifically, he examines the three areas that Goldman and Blanchard (2018) include in their social epistemology: (i) the social interaction of agents and the locus of justification, (ii) the study of groups as the possessors of opinion and knowledge, and (iii) the knowledge-producing consequences of social arrangements and institutions. Winsberg develops interesting questions in all three branches, such as the possible deductions that non-experts can make before a consensus of specialists, the value of dissidence in research, or the nature of epistemic authority in such a plural and distributed science. Maybe it is not the field where Winsberg is a specialist, but he tackles questions of great projection, especially taking into account the external context particularities which permeate the current climate sciences.

To sum up, the work outlined here is a compendium of the problems, discussions, and positions that Winsberg himself has carried out in recent years. It may not be a complete introduction for expert readers, but I think it may be a collection of solidly grounded notions and explanations to begin with in the philosophical study of climate science. If the reader is looking for a rigorous and cutting-edge study, it is possible that some chapters will be presented with little depth for him, with brief descriptions of the problem and little developed arguments. However, it may be useful for researchers who wish to approach for the first time the philosophy of a particular science that is socially very relevant today. For the latter case, there are chapters that can be difficult to understand without having a minimum knowledge-base on the subject, so it is advisable to have a base on general questions of the philosophy of science. For this reason, I believe that, in summary, this book can serve as a good initiation to the epistemology of the climate sciences for the reader who knows classical questions of the philosophy of science, but perhaps not at all recommendable for someone who does not already possess a basic understanding of such themes. If the reader is not an expert in this sub-field of the philosophy of science, I think it is a highly recommended book. As Winsberg himself states, this is a philosophy of a particular science, which emerged a few years ago and is now experiencing a very rapid growth. An area where epistemologists can offer valuable insight to society, as well as to other disciplines involved in elucidating and clarifying complex and novel concepts. Winsberg invites the rest of the philosophers of science who deal with adjacent subjects, advocating the need to present the fundamentals of these sciences in the light of the perennial approaches they have traditionally dealt with in our discipline. 


\section{References}

Duhem P (1991) The Aim and Structure of Physical Theory. Princeton, NJ: Princeton University Press.

Goldman A and Blanchard T (2018) Social Epistemology. In: Zalta E N(ed) The Stanford Encyclopedia of Philosophy (Summer 2018 Edition). Available at: https://plato.stanford.edu/archives/sum2018/entries/ epistemology-social/ (accessed 13.11.2019).

Quine W W (1953) From a Logical Point of View. Cambridge, MA: Harvard University Press.

Suppes P (1969) Models of Data. In: Suppes P (ed) Studies in the Methodology and Foundations of Science: Selected Papers from 1951 to 1969. Dordrecht: Reidel, pp. 24-35.

Winsberg E (2010) Science in the Age of Computer Simulation. Chicago: University of Chicago Press. 